\title{
Puntaje de Gleason en cáncer de próstata: Correlación de la biopsia por punción y prostatectomía radical
}

\author{
OSCAR TAPIA E., ENRIQUE BELLOLIO J., JUAN CARLOS ROA S., PABLO \\ GUZMÁN G., MIGUEL VILLASECA H., JUAN CARLOS ARAYA O.
}

\section{Concordance between Gleason scores of trans rectal biopsies and the surgical piece of radical prostatectomy}

Background: Gleason pathological score in prostate cancer is an important prognostic indicator. However, the concordance between the score of trans rectal needle biopsies and the final score of the surgical piece may be variable. Aim: To analyze the concordance between Gleason scores of trans rectal prostate biopsies and those of the surgical piece obtained after prostatectomy. Material and Methods: Retrospective analysis of 168 pathological records of radical prostatectomies, performed between 1993 and 2009. All these patients had also a trans rectal biopsy performed previously. Patients with less than 12 tissue cylinders obtained during the trans rectal biopsy or incomplete data were not included in this analysis. Results: Sixty eight percent of trans rectal biopsies had Gleason scores that were concordant with those of the surgical piece. The score was higher or lower in 27 and $10 \%$ of biopsies, respectively. Conclusions: Gleason scores of trans rectal biopsies and those of the surgical piece were concordant in $68 \%$ of cases in this series of pathological records.

(Rev Med Chile 2011; 139: 171-176).

Key words: Biopsy, needle; Pancreatic neoplasms; Prostatectomy
Departamento de Anatomía Patológica. Facultad de Medicina. Universidad de La Frontera. Temuco, Chile. Scentifical and Technological Bioresources Nucleus (BIOREN)

Recibido el 2 de marzo de 2010, aceptado el 28 de diciembre de 2010 .

Correspondencia a: Dr. Oscar Tapia E. Departamento de Anatomía Patológica, Facultad de Medicina, Universidad de La Frontera.

Manuel Montt 112. Fono: 045-296530. Código Postal 478-1176. Temuco, Chile. E-mail: otescalona@gmail.com
E stá ampliamente documentado que el grado histológico tumoral constituye un importante factor pronóstico y para la toma de decisiones terapéuticas en pacientes con adenocarcinomas de la próstata, dada su buena correlación con el compromiso tumoral ganglionar linfático, recurrencia local y metástasis a distancia ${ }^{1-6}$. Numerosos han sido los sistemas de clasificación propuestos para el cáncer de próstata, sin embargo, el diseñado por Gleason es el de mayor aceptación a nivel internacional; con una buena correlación inter e intra-observador ${ }^{7,8}$. En su trabajo original, Gleason observó una reproducibilidad entre $50 \%$ y $80 \%$, fluctuando el índice $k$ de concordancia entre 0,14 y 0,502 en las distintas series publicadas ${ }^{9-11}$. Este sistema está basado en la determinación del grado de diferenciación glandular del tumor con- siderando para ello 5 patrones ( 1 al 5 ) y donde el puntaje de Gleason está dado por la sumatoria de los dos patrones predominantes en el tumor. Para su graduación, el patólogo debe observar la arquitectura de la neoplasia utilizando objetivos de bajo aumento ( $4 \mathrm{x}$ o $10 \mathrm{x}$ ), pudiendo utilizar objetivos de aumento mayor para visualizar detalle celular y certificar el diagnóstico, pero no para establecer el $\operatorname{grado}^{7,12,13}$.

Junto al grado histológico, los niveles séricos de antígeno prostático específico (APE) y estadio clínico constituyen algunas de las variables pre-operatorias más importantes que permiten determinar el estado tumoral y predecir el comportamiento biológico del tumor; y de esta manera decidir terapias adecuadas para cada paciente ${ }^{4,6,14-17}$. 
La obtención de muestras para examen histopatológico tiene por finalidad confirmar la sospecha clínica o de laboratorio de cáncer y permitir realizar la adecuada graduación histológica del tumor. Cabe mencionar la importancia de la representatividad de las muestras obtenidas por punción en las cuales se realiza la graduación, existiendo para ello múltiples esquemas y número de muestras propuestos de manera de permitir la mejor correlación entre el puntaje de Gleason de la biopsia de próstata transrectal (BPT) y pieza de prostatectomía $a^{9,18-20}$.

El objetivo de este estudio es evaluar la correlación del puntaje de Gleason de la BPT y pieza de prostatectomía radical.

\section{Material y Método}

Se seleccionaron en forma retrospectiva y consecutiva desde los archivos de la Unidad de Anatomía Patológica del Hospital Hernán Henríquez Aravena de Temuco, 168 casos de prostatectomías radicales entre enero/1993 y julio/2009 con diagnóstico de adenocarcinoma, todas ellas con BPT previa diagnóstica de cáncer. Fueron excluidos del estudio aquellos casos con datos incompletos o sin disponibilidad de una de las biopsias (punción o prostatectomía), casos en los cuales la BPT consideró menos de 12 cilindros de tejido prostático, casos en los cuales el diagnóstico fue realizado en forma incidental en pacientes operados por patología benigna y pacientes con diagnóstico de cáncer que recibieron algún tipo de tratamiento previo a la prostatectomía.

Las muestras obtenidas por punción fueron tomadas por sextantes con aguja de 18 gauge con un mínimo de 12 muestras por paciente. La toma de muestras por punción se realizó en forma digito-dirigida para lesiones palpables sospechosas, mientras que para sujetos con elevación anormal del APE sin lesiones palpables, esta se efectuó en forma ciega entre los años 1993-1998 y a partir del año 1999 guiada por ecografía transrectal.

La pieza de prostatectomía radical se examinó en forma completa y seriada de acuerdo a los protocolos establecidos para el estudio del cáncer prostático ${ }^{21}$.

El examen histopatológico de las BPT consideró por cada inclusión 2 láminas histológicas con tinción de hematoxilina-eosina y 1 con tinción de azul alcián para mucinas ácidas. El estudio de muestras de la pieza de prostatectomía se realizó con tinción de hematoxilina-eosina y desgaste en caso necesario. Todas las biopsias incluidas en el estudio fueron examinadas por el mismo grupo de patólogos (JA, MV, JR) bajo criterios similares.

La graduación histológica del tumor se realizó con el sistema de Gleason, el cual considera 5 patrones de diferenciación glandular ( 1 al 5) siendo el 1 bien diferenciado y 5 poco diferenciado. El puntaje de Gleason constituye la sumatoria de dos patrones morfológicos presentes en el tumor, mientras que para la BPT se considera el patrón $1^{\text {ario }}$ predominante y el más indiferenciado presente independientemente de su extensión; en la prostatectomía el reporte considera el patrón $1^{\text {ario }}$ y $2^{\text {rio }}$ predominantes en el tumor, debiendo este último representar más de $5 \%$ del tumor ${ }^{22,23}$. Teniendo en cuenta estas diferencias y con la finalidad de controlar este sesgo de clasificación; analizamos la graduación de Gleason tanto en la BPT y prostatectomía de acuerdo a los 2 patrones $\left(1^{\text {ario }}\right.$ y $\left.2^{\text {ario }}\right)$ predominantes en el tumor, debiendo el $2^{\text {ario }}$ representar más de $5 \%$; en caso contrario se informa el patrón predominante como $1^{\text {ario }}$ y $2^{\text {ario }}$.

El análisis consideró la correlación del puntaje de Gleason de la BPT y pieza de prostatectomía radical de cada paciente, definiendo correlación en base a concordancia, sobregraduación y subgraduación en el puntaje de Gleason. En forma secundaria, se analizaron también los mismos parámetros por grupo de pacientes en los cuales la BPT se efectuó o no guiada por ecografía transrectal.

\section{Análisis estadístico}

Para el análisis de los resultados se utilizó el programa estadístico Stata 9.2, obteniéndose kappa con su error estándar e intervalo de confianza del 95\% (IC 95\%). La comparación intergrupo siguió el procedimiento sugerido por Cohen en comparación de proporciones. El estudio de concordancia genera el índice kappa, que se divide en tramos de 0 a 1 y se interpretan como: pobre $(0,00$ a 0,20$)$, regular $(0,21$ a 0,40$)$, buena $(0,41$ a 0,60$)$, substancial $(0,61$ a 0,80$)$ y casi perfecta o perfecta $(0,81$ a 1,00$)$.

\section{Resultados}

La mediana de edad en el grupo estudiado fue 67 años (40-84 años).

El 38\% (64) de las biopsias por punción co- 
Tabla 1. Correlación del puntaje de Gleason entre la BPT y prostatectomía

\begin{tabular}{|cccccccccc|}
\hline $\begin{array}{c}\text { Puntaje de } \\
\text { Gleason } \\
\text { punción }\end{array}$ & $\mathbf{8}$ & $\mathbf{7}$ & \multicolumn{7}{c|}{$\begin{array}{c}\text { Puntaje de Gleason } \\
\text { Prostatectomía }\end{array}$} \\
3 & $\mathbf{7}$ & $\mathbf{5}$ & $\mathbf{6}$ & $\mathbf{7}$ & $\mathbf{8}$ & $\mathbf{9}$ & $\mathbf{1 0}$ & Total \\
4 & $(0) /[1]$ & $(0) /[0]$ & $(0) /[0]$ & $(0) /[0]$ & $(0) /[0]$ & $(0) /[0]$ & $(0) /[0]$ & $(0) /[0]$ & $(0) /[1]$ \\
5 & $(0) /[0]$ & $(0) /[0]$ & $(0) /[0]$ & $(1) /[0]$ & $(0) /[0]$ & $(0) /[0]$ & $(0) /[0]$ & $(0) /[0]$ & $(1) /[0]$ \\
6 & $(0) /[0]$ & $(0) /[0]$ & $(3) /[7]$ & $(1) /[1]$ & $(2) /[3]$ & $(0) /[0]$ & $(0) /[0]$ & $(0) /[0]$ & $(6) /[11]$ \\
7 & $(0) /[0]$ & $(0) /[0]$ & $(1) /[1]$ & $(14) /[16]$ & $(12) /[14]$ & $(0) /[0]$ & $(0) /[1]$ & $(0) /[0]$ & $(27) /[32]$ \\
8 & $(0) /[0]$ & $(0) /[0]$ & $(0) /[0]$ & $(3) /[2]$ & $(24) /[28]$ & $(2) /[1]$ & $(2) /[2]$ & $(0) /[0]$ & $(31) /[33]$ \\
9 & $(0) /[0]$ & $(0) /[0]$ & $(0) /[0]$ & $(0) /[1]$ & $(2) /[4]$ & $(1) /[2]$ & $(0) /[3]$ & $(0) /[0]$ & $(3) /[10]$ \\
10 & $(0) /[0]$ & $(0) /[0]$ & $(0) /[0]$ & $(0) /[0]$ & $(0) /[1]$ & $(0) /[0]$ & $(4) /[3]$ & $(1) /[0]$ & $(5) /[4]$ \\
Total & $(0) /[0]$ & $(0) /[0]$ & $(0) /[0]$ & $(0) /[0]$ & $(0) /[0]$ & $(0) /[0]$ & $(1) /[0]$ & $(1) /[2]$ & $(2) /[2]$ \\
\hline
\end{tabular}

* (BBP dígito-dirigida)/[BBP eco-dirigida] Concordancia Global: Kappa $=0,47 \quad$ Kappa ponderado $=0,61$ (BPP No eco-dirigida): Kappa $=0,44 \quad$ Kappa ponderado $=0,60$ [BPP eco-dirigida]: $\quad$ Kappa $=0,49 \quad$ Kappa ponderado $=0,62$

Tabla 2. Correlación global y por grupos de acuerdo al puntaje de Gleason

\begin{tabular}{|c|c|c|c|}
\hline Correlación & Global & BPT No eco-dirigida & BPT eco-dirigida \\
\hline Concordancia & $106 / 16863 \%$ & $47 / 7563 \%$ & $59 / 9363 \%$ \\
\hline Subgraduación & $46 / 168 \quad 27 \%$ & $21 / 7528 \%$ & $25 / 93 \quad 27 \%$ \\
\hline Sobregraduación & $16 / 16810 \%$ & $7 / 759 \%$ & $9 / 93 \quad 10 \%$ \\
\hline
\end{tabular}

rrespondió a adenocarcinomas con un puntaje de Gleason 7 y 35\% (59) puntaje de Gleason 6. En la piezas de prostatectomía el 54\% (90) y 23\% (39) correspondió a carcinomas con puntaje 7 y 6 respectivamente (Tabla 1 ).

El nivel de correlación para el puntaje de Gleason de los 168 casos estudiados mostró concordancia, subgraduación y sobregraduación en $63 \%, 27 \%$ y $10 \%$ respectivamente con un índice kappa ponderado de 0,61 (Tablas 1 y 2). El análisis de concordancia por separado de los grupos con BPT sin y con eco-dirigida demostró un índice kappa ponderado de 0,60 y 0,62 respectivamente (Tabla 1) con un porcentaje de concordancia de $63 \%$ para sujetos con y sin BPT (Tabla 2).

El porcentaje promedio de tumor encontrado en la biopsia por punción y prostatectomía fue $41,8 \%$ y $23,3 \%$ respectivamente, siendo el peso promedio de la pieza quirúrgica 54,6 g.
Para los casos correlacionados el peso promedio de la pieza quirúrgica de prostatectomía, porcentaje promedio de tumor encontrado en la biopsia por punción y prostatectomía fue 53,1 g, $45,3 \%$ y $20,9 \%$ respectivamente.

Para los casos con discordancia el peso promedio de la pieza quirúrgica de prostatectomía, porcentaje promedio de tumor encontrado en la biopsia por punción y prostatectomía fue 57,2 g, $35,8 \%$ y $27,4 \%$ respectivamente.

\section{Discusión}

Es ampliamente reportado que el grado histológico tumoral es uno de los factores pronósticos independientes más importante en pacientes con adenocarcinomas primarios de la próstata. Una graduación adecuada y reproducible es de vital 
importancia, pues el puntaje de Gleason presenta una directa relación con invasión capsular y de vesículas seminales, compromiso ganglionar linfático y de márgenes quirúrgicos; lo que permite junto a otras variables predecir adecuadamente el comportamiento biológico del tumor expresado como recurrencia local, metástasis a distancia y supervivencia ${ }^{1-6}$.

Nuestros resultados muestran una buena correlación entre el puntaje de Gleason de la biopsia por punción y el de la pieza de prostatectomía radical con una concordancia de $63 \%$ similar a la obtenida en la series reportadas por Catalona et al y Spires et al, entre otros ${ }^{25-27}$, sin observar diferencias estadísticas significativas entre los grupos en los cuales la BPT se realizaba o no guiada por ecografia transrectal. El puntaje de Gleason fue subestimado en $27 \%$ de los casos, encontrándose dentro de los resultados reportados, pero muy por debajo de lo publicado por otros autores que describen cifras de hasta 54\% de subgraduación del puntaje de Gleason en biopsias por punción ${ }^{9,26-30}$. Esta baja proporción de subgraduación en relación a otras series, se explica por la existencia de sólo dos biopsias por punción diagnosticadas como adenocarcinomas bien diferenciados (Gleason 2-4), lo que concuerda con la recomendación publicada por Epstein quien propone no asignar puntajes de Gleason 2, 3 ó 4 en biopsias por punción de manera de reducir la posibilidad de subgraduación de tumores que generalmente son pequeños y ubicados en la zona de transición ${ }^{(31-33)}$.

Si bien en nuestra serie tenemos adecuados niveles de concordancia tanto en forma global para el grupo de estudio y en forma separada para los grupos con y sin BPT eco-dirigida, la subgraduación es una de los principales dificultades, mencionando la literatura como principales posibles causas de error en la graduación histológica a la obtención de muestras poco o no representativas de la lesión, y donde según algunos autores, la obtención de una mayor cantidad de tejido aplicando protocolos que consideran un mayor número de toma de muestras, permitiría controlar esta variable producto de la condición de heterogeneidad tumoral; aun cuando existen reportes en los cuales la mejoría en la correlación es despreciable al comparar esquemas por punción que consideran diferentes números de toma de muestra ${ }^{9,34-36}$. El otro elemento a considerar es la correcta interpretación por parte del anatomo- patólogo, existiendo trabajos que demuestran una mejor correlación cuando la graduación es realizada por patólogos especialistas en el área y tanto biopsia por punción como quirúrgica son examinados por el mismo patólogo o grupo de patólogos, suponiendo esto último la existencia de difusión de consensos locales y aplicación de protocolos validados en este tema ${ }^{9,29,35-39}$. En nuestra serie, al analizar y comparar el grupo de tumores con puntaje de Gleason subgraduados y sin discordancia constatamos en los casos subgraduados un menor porcentaje promedio de tumor reportado en la biopsia por punción $(35,89 \%$ vs $45,38 \%$; $<<0,05)$ y un mayor porcentaje promedio de tumor reportado en la pieza quirúrgica $(27,4 \%$ vs $20,9 \%$; $p<0,001$ ); en base a estos resultados la no concordancia en estos casos la atribuimos a la poca representatividad de la muestras obtenidas por punción. Por otro lado, en los 16 casos sobregraduados se revisaron las láminas histológicas de la biopsia por punción y prostatectomía, en 7 casos la discordancia se atribuyó a un error de interpretación del patrón de Gleason mientras que en los otros 9 casos se observó que uno de los patrones informado en la biopsia por punción al momento de procesar la pieza quirúrgica no constituye un patrón predominante por lo que no es reportado en el informe final.

Aun cuando el nivel de correlación estadístico en nuestra serie es substancial, los resultados de la biopsia por punción deben ser interpretados cuidadosamente teniendo en cuenta las limitaciones propias del procedimiento de toma de muestra e interpretación de la biopsia, sin embargo, adecuados niveles de correlación como los obtenido en nuestra serie permiten al clínico-oncólogo ofrecer alternativas terapéuticas adecuadas a perfiles tumorales específicos ${ }^{17,40}$.

\section{Referencias}

1. Narain V, Bianco FJ Jr, Grignon DJ, Sakr WA, Pontes JE, Wood DP Jr. How accurately does prostate biopsy Gleason score predict pathologic findings and disease free survival?. Prostate 2001; 49: 185-90.

2. Feneley MR, Partin AW. Indicators of pathologic stage of prostate cancer and their use in clinical practice. Urol Clin North Am 2001; 28: 443-58.

3. Derweesh IH, Kupelian PA, Zippe C, Levin HS, Brainard J, Magi-Galluzi C, et al. Continuing trends in pathologi- 
cal stage migration in radical prostatectomy specimens. Urol Oncol 2004; 22: 300-6.

4. Stamey T, Caldwell M, Mcneal J, Molley R, Hermandez M, Downs J. The Prostate Specific Antigen era in the United States is over for Prostate Cancer: What happened in the last 20 years? J Urol 2002; 172: 1297-301.

5. Bostwick DG, Grignon DI, Hammond EH, Amin MB, Cohen M, Crawford D, et al. Prognostic factors in prostate cancer: College of American Pathologist consensus statement 1999. Arch Pathol Lab Med 2000; 124: 9951000.

6. Partin AW, Kattan MW, Subong EN, Walsh PC, Wojno $\mathrm{KJ}$, Oesterling JE, et al. Combination of prostate-specific antigen, clinical stage, and Gleason score to predict pathological stage of localized prostate cancer: A multiinstitutional update. JAMA 1997; 277: 1445-51.

7. Gleason DF, Mellinger GT. Prediction of prognosis for prostatic carcinoma by combined histological grading and clinical staging. J Urol 1974; 111: 58-64.

8. Bostwick DG. Grading prostate cancer. Am J Clin Pathol 1994; 102: S38-56.

9. Cookson MS, Fleshner NE, Soloway SM, Fair WR. Correlation between Gleason score of needle biopsy and radical prostatectomy specimen: accuracy and clinical implications. J Urol 1997; 157: 559-62.

10. Djavan B, Kadesky K, Klopukh B, Marberger M, Roehrborn CG. Gleason scores from prostate biopsies obtained with 18-gauge biopsy needles poorly predict Gleason scores of radical prostatectomy specimens. Eur Urol 1998; 33: 261-70.

11. King CR. Patterns of prostate cancer biopsy grading: trends and clinical implications. Int J Cancer 2000; 90: 305-11.

12. Gleason DF. Classification of prostatic carcinomas. Cancer Chemoter Rep 1966; 50: 125-8.

13. Gleason DF. Histologic grading and clinical staging of prostatic carcinoma. Urologic Pathology: The Prostate, M Tannenbaum, ed. Lea and Feibiger: Philadelphia.

14. Scherr D, Swindle PW, Scardino PT and National Comprehensive Cancer Network. National Comprehensive Cancer Network Guidelines for the Management of Prostate Cancer. Urology Suppl 2003; 61: 14-24.

15. D'amico V, Whittington R, Schultz D. Outcome based staginng for clinically localized adenocarcinoma of the prostate. J Urol 1977; 158: 1422-5.

16. Badalamnt RA, Millaer MC, Peller PA. An algorithm for predicting nonorgan confined prostate cancer using the results obtained from sextant core biopsies with prostate specific antigen level. J Urol 1996; 156: 1375-9.

17. Djavan B, Kadesky K, Klopukh B. Las puntuaciones de Gleason obtenidas en biopsia por punción muestran poca correlación con la pieza de prostatectomía radical. Eur Urol 1999; 6: 369-72.

18. Matlaga BR, Eskew LA, Mccullough DL. Prostate biopsy: indications and technique. J Urol 2003; 169: 12-9.

19. Grossklaus DJ, Coffey CS, Shappell SB, Jack GS, Cookson MS. Prediction of tumor volume and pathological stage in radical prostatectomy specimens is not improved by taking more prostate needle-biopsy cores. BJU Int 2001; 88: 722-6.

20. San Francisco IF, Dewolf WC, Rosen S, Upton M, Olumi AF. Extended prostate needle biopsy improves concordance of Gleason grading between prostate needle biopsy and radical prostatectomy. J Urol 2003; 169: 136-40.

21. True LD. Surgical pathology examination of the prostate gland. Practice survey by American society of clinical pathologists. Am J Clin Pathol 1994; 102: 572-9.

22. Epstein JI, Allsbrook WC Jr, Amin MB, Egevad LL. ISUP grading committee. The 2005 International Society of Urologic Pathology (ISUP) Consensus Conference on Gleason grading of Prostatic Carcinoma. Am J Surg Pathol 2005; 29: 1228-42.

23. Billis A, Guimaraes MS, Freitas LL, Meirelles L, Magna LA, Ferreira U. The impact of the 2005 international society of urological pathology consensus conference on standard Gleason grading of prostatic carcinoma in needle biopsies. J Urol 2008; 180: 548-52.

24. Young RH, Srigley JR, Amin MB, Ulbright TM, Cubilla A. Tumors of the Prostate Gland, Seminal Vesicle, Male Urethra and Penis. Washington, DC: Armed Forces Institute of Pathology; 2000. Atlas of Tumor Pathology. 3rd series, fascicle 28.

25. Catalona WI, Stein AI, Fair WR. Grading errors in prostatic needle biopsies: relation to the accuracy of tumor grade in predicting pelvic lymph node metastases. J Urol 1982; 127: 919-22.

26. Spires SE, Cibull Ml, Wood DP, Miller S, Spires SM, Banks ER. Gleason histologic grading in prostatic carcinoma. Correlation of 18 gauge core biopsy with prostatectomy. Arch Pathol Lab Med 1994; 118: 705-8.

27. Rajinikanth A, Manoharan M, Soloway C, Civantos F, Soloway M. Trends in Gleason Score: Concordance Between Biopsy and Prostatectomy over 15 Years. Urology 2008; 72: 177-82.

28. Bostwick DG. Gleason grading of prostatic leedle biopsies. Correlation with grade in 316 matched prostatectomies. Am J Surg Pathol 1994; 18: 796-803.

29. Arellano L, Castillo O, Metrebian E. Concordancia diagnóstica del puntaje de Gleason en biopsia por punción y prostatectomía radical y sus consecuencias clínicas. Rev Med Chile 2004; 132: 971-8.

30. Kvåle R, Møller B, Wahlqvist R, Fosså SD, Berner A, 
Puntaje de Gleason en cáncer de próstata - O. Tapia et al

Busch C, Kyrdalen AE, Svindland A, Viset T, Halvorsen OJ. Concordance between Gleason scores of needle biopsies and radical prostatectomy specimens: a population-based study. BJU Int 2009; 103: 1647-54.

31. Campbell's Urology. Octava Edición Capítulo 86. Patología de la Neoplasia prostática. Epstein, JI.

32. Epstein JI, Potter SR. The pathological interpretation and significance of prostate needle biopsy findings: implications and current controversies. J Urol 2001; 166: 402-10.

33. Epstein JI. Gleason score 2-4 adenocarcinoma of the prostate on needle biopsy: a diagnosis that should not be made. Am J Surg Pathol 2000; 24: 477-8.

34. Egevad L, Norlen BJ, Norberg M. The value of multiple core biopsies for predicting the Gleason score of prostate cancer. BJU Int 2001; 88: 716-721.

35. King CR, Long JP. Prostate biopsy grading errors: a sampling problem?. Int J Cancer 2000; 90: 326-30.

36. Lattouf JB, Saad F. Gleason score on biopsy: is it reliable for predicting the final grade on pathology? BJU Intern 2002; 90: 694-8.

37. Allsbrook WC, Mangold KA, Johnson MH, Lane RB, Lane CG, Amin MB, et al. Interobserver reproducibility of Gleason grading of prostatic carcinoma: urologic pathologists. Hum Pathol 2001; 32: 74-88.

38. Mikami Y, Manabe T, Epstein JI, Shiraishi T, Furusato $\mathrm{M}$, Tsuzuki $\mathrm{M}$, et al. Accuracy of Gleason grading by practicing pathologists and the impact of education on improving agreement. Hum Pathol 2003; 34: 658-65.

39. Grossfeld GD, Chang JI, Broering JM, Li YP, Lubeck DP, Flanders $\mathrm{S}$, et al. Under staging and under grading in a contemporary series of patients undergoing radical prostatectomy: results from the cancer of the prostate strategic urologic research endevon database. J Urol 2001; 165: 851-6.

40. Prosa J, Gross N, Bastide C. Correlation between Gleason score of prostatic biopsies and the one of the radical prostatectomy specimen. Prog Urol 2001; 111: 45-8. 